\title{
Ocular palsy associated with aggressive NK-cell leukemia
}

\author{
Masaharu Tsubokura · Takuya Yamashita · \\ Shun-ichiro Kageyama • Ikuyo Endo • \\ Hiromasa Tsuda $\cdot$ Hideki Akiyama
}

Received: 2 March 2011 / Revised: 28 April 2011/Accepted: 6 May 2011/Published online: 25 May 2011

(c) The Japanese Society of Hematology 2011

\begin{abstract}
A 22-year-old woman was referred to our hospital with a 1-month history of recurrent fever, subacute onset of bilateral orbital pain, and diplopia. Physical examination revealed the limits of supraduction of her right eye and infraduction of her left eye. A bone marrow aspirate from the ilium showed scattered large immature-looking lymphocytes $(15.8 \%)$ with pale cytoplasm, fine nuclear chromatin, and nucleoli (Fig. 1). These cells were stained positively with CD56, TIA-1, granzyme, and perforin, and were stained negatively with CD3. In situ hybridization for EBER demonstrated that the cells were EBV-positive. A diagnosis of aggressive NK-cell leukemia was made.

Magnetic resonance imaging of orbital cavity showed marked enlargement, and diffuse high intensity signals in the right superior rectus muscle and left inferior rectus muscle on fat-saturated T2-weighted image (Fig. 1). These lesions were diffusely contrast-enhanced on fat-saturated T1-weighted image (Fig. 2).
\end{abstract}

After two courses of dexamethasone, methotrexate, ifosfamide, L-asparaginase, and etoposide chemotherapy,

\footnotetext{
M. Tsubokura $(\bowtie) \cdot$ T. Yamashita $\cdot$ S. Kageyama $\cdot$ H. Akiyama Hematology Division, Tokyo Metropolitan Cancer and Infectious Diseases Center, Komagome Hospital, 3-18-22 Honkomagome, Bunkyo-ku,

Tokyo 113-8677, Japan

e-mail: tsubokura-tky@umin.ac.jp

I. Endo

Department of Radiology, Tokyo Metropolitan Cancer and Infectious Diseases Center,

Komagome Hospital, Tokyo, Japan

H. Tsuda

Department of Neurology, Tokyo Metropolitan Cancer and Infectious Diseases Center,

Komagome Hospital, Tokyo, Japan
}

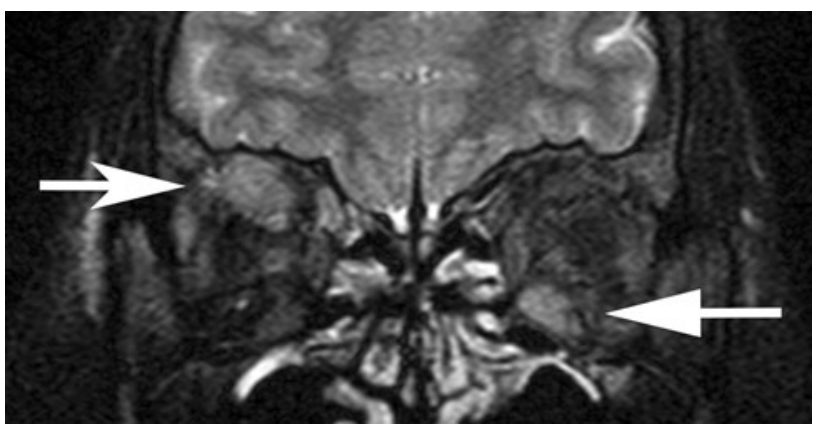

Fig. 1 MRI of orbital cavity showed the marked enlargement, and diffuse high intensity signal in the right superior rectus muscle and left inferior rectus muscle on fat-saturated T2-weighted image

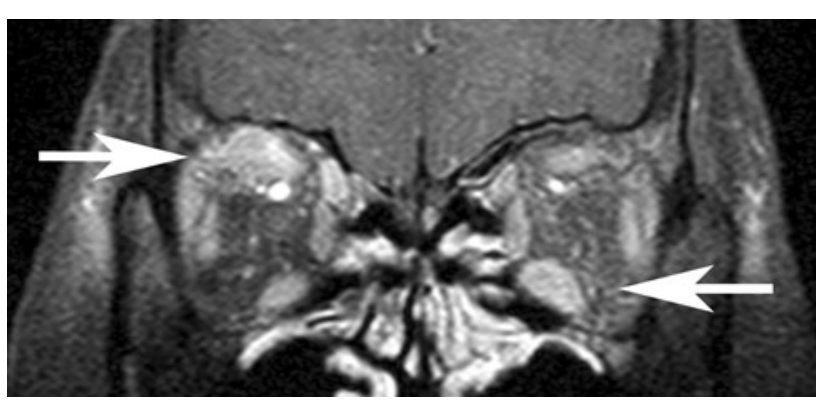

Fig. 2 Eye lesions were diffusely contrast-enhanced on fat-saturated T1-weighted image

she underwent cord blood transplantation with a preparative regimen comprised of etoposide, cyclophosphamide, and total body irradiation. She achieved complete remission, and her ocular manifestations completely resolved.

We could not perform biopsy of the lesions due to the poor general condition of the patient, and the exact etiology 
of her ocular manifestation remains to be elucidated. While her ocular symptoms occurred in parallel to the progression of the leukemia, a number of possibilities for the cause of her ocular involvement can be raised, including tumor infiltration, infection, or paraneoplastic myositis. An accumulation of cases of ocular involvement associated with NK-cell leukemia is required.

Conflict of interest None declared. 